\title{
Dosage de l'ion nitrate en solution nutritive et en présence de polyéthylène glycol par spectrométrie UV
}

\author{
G Vercambre, S Adamowicz * \\ Unité de recherche en écophysiologie et horticulture, site Agroparc, Inra, 84914 Avignon cedex 9, France
}

(Reçu le 15 mars 1994 ; accepté le 29 décembre 1995)

\begin{abstract}
Résumé - Cette étude présente une méthode de dosage du nitrate en solution nutritive basée sur la spectrométrie UV. Applicable à une large gamme de concentration, elle prend en compte les molécules interférentes présentes généralement dans les solutions nutritives. L'étude des caractéristiques spectrales de celles-ci montre que les chélatants du fer et le polyéthylène glycol (PEG), macromolécule utilisée pour simuler des disponibilités hydriques réduites en solution nutritive, présentent une absorbance UV non négligeable. En mesurant les densités optiques à autant de longueurs d'onde qu'il existe de molécules optiquement actives en solution, la concentration de l'ion nitrate est calculée par résolution d'un système d'équations linéaires. La seule contrainte de la méthode consiste à se ramener, par simple dilution dans l'eau, à une concentration où la réponse de l'absorbance est linéaire pour l'ion nitrate. La justesse et la répétabilité de la mesure sont très bonnes pour des intervalles de concentration larges, tant en nitrate qu'en PEG, ouvrant ainsi la possibilité d'étudier précisément la nutrition nitrique en condition d'alimentation hydrique réduite.
\end{abstract}

\section{EDTA / nitrate / polyéthylène glycol / EDDHA / spectrométrie UV}

Summary - Measuring nitrate concentration in nutrient solutions in the presence of polyethylene glycol through UV spectrometry. A simple method has been devised for the determination of nitrate concentration in nutrient solution using UV spectrophotometry. It is suitable for an accurate determination of nitrate in the presence of iron chelates as well as polyethylene glycol (PEG), allowing the study of nitrate nutrition of plants in conditions of reduced water availability. Nitrate has a peak absorbance at $201 \mathrm{~nm}$, independent of $\mathrm{pH}$ and ionic strength, and showing a linear relationship with concentration up to $0.20 \times 10^{-3} \mathrm{~mol} . \mathrm{L}^{-1}$. A comparison has been made between spectral properties of two iron chelates, Fe-EDDHA and Fe-EDTA. It is shown that the latter is more suitable for an accurate determination of nitrate concentration due to its lower specific absorbance and independence of $\mathrm{pH}$ conditions. The spectral properties of PEG are independent of $\mathrm{pH}$ and show a high absorbance in the 190-230 nm range. It is shown that nitrate concentration can be calculated with an accuracy better than $3 \%$, by measuring absorbance at two or three wavelengths (without or with PEG respectively), provided that total absorbance is below 2 and nitrate concentration below $0.20 \times 10^{-3} \mathrm{~mol} . \mathrm{L}^{-1}$. In all cases, this can be reached simply by a mere dilution with water. Due to the non-linearity of PEG absorbance with concentration, a numerical method has been devised to solve the system of three equations.

EDTA / nitrate / polyethylene glycol / EDDHA / UV spectrometry

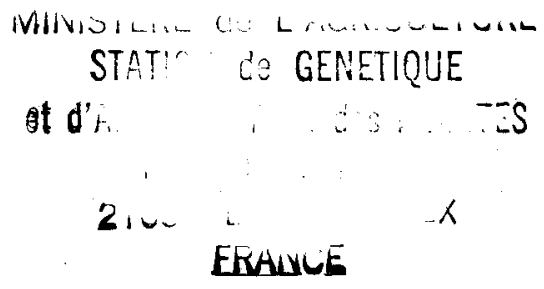




\section{INTRODUCTION}

De très nombreuses méthodes sont applicables au dosage de l'ion nitrate en solution nutritive. Elles peuvent être classées en deux catégories: les méthodes indirectes, où l'on dose une espèce chimique différente de l'ion nitrate, obtenue après une série de réactions, et les méthodes directes qui $n$ 'impliquent aucune transformation de l'ion nitrate. Selon les cas, les contraintes de mise en œuvre et les interférences sont plus ou moins fortes.

Les réactions de nitration ou d'oxydation de composés aromatiques en présence d'acide sulfurique permettent d'obtenir des composés dosables colorimétriquement (Snell et Snell, 1949 ; Charlot, 1961) ou par chromatographie en phase gazeuse (Glover et Hoffsommer, 1974 ; Wu et Saschenbrecker, 1977 ; Quinche et Dvorak, 1980). Les principales interférences sont dues aux composés organiques qui gènent la colorimétrie en raison de leur caramélisation par l'acide sulfurique (Norwitz et al, 1979), et aux chlorures (West et Ramachandran, 1966 ; Lambert et Dubois, 1971) responsables de pertes d'azote sous forme de chlorure de nitrosyle volatile formé par réaction entre les ions nitrate et chlorure en milieu sulfurique (Norwitz et Keliher, 1978). Le dosage colorimétrique, après extraction dans un solvant organique d'un complexe formé entre l'ion nitrate et un colorant cationique, est aussi utilisé dans les eaux (Pokorny et Likussar, 1968 ; Gholse, 1984) et les extraits de sol (Ciesielski et al, 1978). Cependant cette méthode est peu spécifique, de nombreux anions minéraux et organiques pouvant former des complexes stables avec ces cations. Toutes ces méthodes sont coûteuses en temps et requièrent l'utilisation d'un réactif agressif, l'acide sulfurique concentré, ou d'un solvant organique.

La réduction de l'ion nitrate en nitrite ou en ammonium, deux formes d'azote minéral faciles à doser, est la voie chimique la plus suivie. Le dosage colorimétrique de l'ion nitrite, après réaction de Griess, est sensible et spécifique, mais l'étape de réduction nécessite des conditions physicochimiques définies et très stables (Kamphake et al, 1967 ; Smith et Scott, 1983) et se prête surtout au dosage automatique en flux continu. La réduction en ammonium par l'alliage Devarda suivie de sa distillation et de sa titration (Bremner et Keeney, 1965) est peu sensible aux interférents dans les milieux pauvres en azote organique (Adamowicz et al, 1982), mais s'avère lente et fastidieuse.
La potentiométrie, ou utilisation d'électrodes sélectives, permet un dosage direct et rapide de l'ion nitrate, mais sa précision est faible pour les valeurs inférieures à $10^{-3} \mathrm{~mol}^{-1}{ }^{-1}$ (Keeney et al, 1970). Les ions chlorure et les anions organiques interfèrent (Keeney et al, 1970 ; Novozamsky et al, 1983 ; Csiky et al, 1985), rendant nécessaire l'élimination des premiers par précipitation avec un sel d'argent (Talbudeen et Page, 1983) et la complexation des seconds par des ions aluminium (Paul et Carlson, 1968 ; Baker et Smith, 1969). De plus, certaines molécules organiques peuvent aussi détériorer la membrane des électrodes sélectives (Csiky et al, 1985). Le dosage par HPLC (high performance liquid chromatography) a récemment fait l'objet de larges développements. La séparation des molécules sur colonne, suivie de la détection conductimétrique des ions, permet le dosage de très nombreux minéraux, en particulier du nitrate (Williams, 1983 ; Perrone et Gant, 1984). Toutefois, cette technique présente un coût élevé et une cadence très faible (typiquement, 30 minutes par échantillon).

Les solutions nutritives sont un cas particulier des solutions à doser, les éléments présents en solution étant connus. Outre les molécules classiquement présentes en solution nutritive, l'emploi de polyéthylène glycol (PEG) a été récemment développé. Cette macromolécule est utilisée pour simuler des disponibilités hydriques réduites en solution nutritive (Michel, 1983). La forte charge organique apportée par le PEG, ainsi que ses propriétés osmotiques et tensioactives, font obstacle au dosage de l'ion nitrate par les méthodes citées. Des essais préliminaires de dosage du nitrate par HPLC et par colorimétrie automatique après réduction en nitrite sur une chaîne munie d'un dialyseur ont montré que le $P E G$ interfère de façon importante dans les deux cas.

L'ion nitrate présente une forte absorption dans le lointain UV (Buck et al, 1954 ; Altshuler et Wartburg, 1960), initialement mise à profit pour son dosage dans les eaux (Hoather et Rackham, 1959). Bien que les ions $\mathrm{Cl}^{-}, \mathrm{H}_{2} \mathrm{PO}_{2}^{-}$, $\mathrm{HPO}_{3}^{2-}, \mathrm{PO}_{4}^{3-}$ et $\mathrm{SO}_{4}^{2-}$ soient transparents dans I'UV (Perrone et Gant, 1984), la bande d'absorption du nitrate est cependant peu spécifique. Les molécules interférentes ont été plus largement étudiées sur des extraits de sol. Ce sont essentiellement les ions nitreux et ferriques, ainsi que de nombreuses molécules organiques insaturées. Pour réduire l'interférence due aux ions ferriques et aux nitrites, la solution extraite est diluée dans l'acide perchlorique (Bastian et al, 1958 ; Cawse, 1967), méthode adoptée pour des 
études de physiologie végétale (Breteler et al, 1979 ; Breteler et Nissen, 1982 ; Ni et Breevers, 1990 ; Le Bot, 1991 ; Lainé et al, 1993). L'interférence des molécules organiques peut être éliminée préalablement par défécation (Cawse, 1967), ou déduite en mesurant la différence d'absorbance UV avant et après destruction des ions nitrate (Armstrong, 1963), ou enfin prise en compte par un coefficient de correction empirique moyen (Heanes, 1982).

Dans cet article, nous nous proposons de simplifier la méthode utilisant la spectrométrie UV, de l'adapter au cas particulier des solutions nutritives, et de la rendre compatible avec la présence de PEG. La simplification porte sur la suppression de tout réactif, en particulier de l'acide perchlorique. En effet, l'usage de cet artifice diminue mais ne supprime pas les interférences. Nous préférons étudier les caractéristiques spectrales des substances interférentes identifiées (chélates de fer et PEG) afin de mettre au point une méthode de dosage directe qui prenne en compte leur contribution effective à l'absorbance UV dans le domaine spectral où l'ion nitrate est dosé. Dans les solutions nutritives, le fer est toujours apporté sous forme de chélate. Nous en avons comparé deux parmi les plus utilisés, I'EDDHA et I'EDTA, afin de choisir la forme la plus favorable à un dosage précis du nitrate. Ceci implique que les expérimentations de nutrition minérale ultérieures devront tenir compte de ce choix. Après une étude détaillée des spectres de chacun des composés pris isolément, ion nitrate, chélates de fer et PEG, nous proposons une méthode simple du dosage de leur mélange, qui permet en particulier d'aborder les problèmes de nutrition nitrique en condition de disponibilité hydrique réduite.

\section{MATÉRIEL ET MÉTHODES}

Une solution mère de nitrate de sodium $510^{-3}$ mol. $\mathrm{L}^{-1}$ est préparée à partir d'une solution standard Orion 0,1 mol. $L^{-1}$ (à $1 \%$ près), dont on a vérifié la pureté par

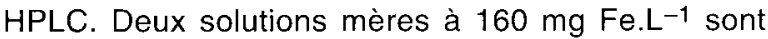
fabriquées, l'une à partir d'EDDHA-Fe (séquestrène à $6 \%$ de fer, Ciba-Geigy, lot $n^{\circ} 073 S, 1991$ ), l'autre à partir d'EDTA (EDTA, sel Fe-Na, $1 \mathrm{H}_{2} \mathrm{O}$ mol.mol-1, Sigma, lot $\left.n^{\circ} 80 \mathrm{H} 0838,1992\right)$. Dans les solutions nutritives, les concentrations en fer sont généralement comprises entre 0,6 et $3,2 \mathrm{mg} \mathrm{Fe} \cdot \mathrm{L}^{-1}$. Des solutions à différentes concentrations en polyéthylène glycol 6000 (PEG 6000, Prolabo, lot $n^{\circ} 291 \mathrm{AP}, 1993$ ) ont également été réalisées. Les concentrations des solutions sont exprimées en g.g ${ }^{-1}$ (PEG/eau). Le poids moléculaire moyen du PEG, $6000 \mathrm{~g}$ par mole, a été choisi pour, d'une part empêcher son absorption par la plante et éviter d'éventuels effets toxiques et, d'autre part, obtenir une solution la plus homogène possible. L'utilisation de PEG de très fort poids moléculaire produit une solution moussant très facilement, donc hautement hétérogène. Pour les solutions de PEG, l'ajustement de Michel (1983) permet de déterminer le potentiel hydrique en fonction de la température et de la concentration en PEG. En pratique, ceci nous a conduit à utiliser des concentrations en PEG de 0,12 à $0,17 \mathrm{~g} \cdot \mathrm{g}^{-1}$

Pour étudier l'influence de la force ionique et du pH sur les caractéristiques spectrales des corps étudiés, nous avons préparé des tampons $\mathrm{pH}, \mathrm{H}_{3} \mathrm{PO}_{4} /$ $\mathrm{NaH}_{2} \mathrm{PO}_{4} / \mathrm{NaOH}$, de force ionique $2510^{-3} \mathrm{~mol}^{-1}$, dans une gamme comprise entre 3,5 et 7 unités $\mathrm{pH}$.

Toutes les solutions sont réalisées avec de l'eau ultrapure (Milli-Q Plus, Millipore SA). Les dilutions ont été faites avec un diluteur automatique Gilson (Modèle 401), équipé d'un piston de $5 \mathrm{~mL}$.

Un spectromètre Perkin-Elmer Lambda2 (donné pour linéaire jusqu'à une densité optique de 3,5) avec logiciel PECSS a été utilisé, avec les réglages suivants : bande passante $2 \mathrm{~nm}$, temps de réponse 1 , correspond à environ 3 secondes. Les cuves en quartz (Hellma QS) ont un trajet optique de $10 \mathrm{~mm}$. Outre les longueurs d'onde caractéristiques des spectres étudiés $(201,257$ et $280 \mathrm{~nm})$, nous avons systématiquement mesuré les absorbances à 206, 214 et $226 \mathrm{~nm}$, valeurs standard des spectromètres à filtre.

Pour un corps pur, la loi de Beer-Lambert relie sa concentration à l'absorbance à une longueur d'onde donnée selon la relation :

$$
\mathrm{DO}=\varepsilon \times \mathrm{I} \times \mathrm{C}
$$

avec DO : densité optique, I : longueur du trajet optique $(\mathrm{cm}), \varepsilon$ : coefficient d'extinction, $\mathrm{c}$ : concentration (en molarité).

Le coefficient d'extinction est une constante physique caractéristique du corps étudié et de la longueur d'onde. Lorsqu'il est connu, on utilise généralement cette relation pour calculer la concentration à partir d'une mesure de densité optique.

Dans un mélange, en l'absence d'interaction entre les molécules en solution, les absorbances sont additives:

$$
\mathrm{DO}_{\lambda}^{\text {solution }}=\sum_{i} \mathrm{DO}_{\lambda}^{\mathrm{Xi}}
$$

avec $\mathrm{DO}_{\lambda}^{\text {solution }}$ : absorbance de la solution, à la longueur d'onde $\lambda, D O_{\lambda}^{x_{i}}$ : absorbance due à la molécule $i$.

En utilisant la formule de Beer-Lambert, cette équation devient, avec I $=1 \mathrm{~cm}$ :

$$
\mathrm{DO}_{\lambda}^{\text {solution }}=\sum_{i} \varepsilon_{\lambda}^{\mathrm{Xi}} \times[X i]
$$

avec $\varepsilon_{\lambda}^{\mathrm{Xi}}$ : coefficient d'extinction de la molécule $i$, à la longueur d'onde $\lambda,[X i]$ : concentration de la molécule $i$. 
Soit une solution contenant $n$ molécules optiquement actives, dont on a déterminé préalablement les coefficients d'extinction à $n$ longueurs d'onde. En mesurant la densité optique de la solution aux $n$ longueurs d'onde, on établit un système de $n$ équations à $n$ inconnues, les concentrations recherchées. Dans la mesure où ce système n'est pas indéterminé, condition réalisée si les contributions à l'absorbance totale de chaque molécule varient pour les $n$ longueurs d'onde, sa résolution permet de calculer la concentration de chaque molécule. L'objectif de ce travail est de rechercher la meilleure combinaison de $n$ longueurs d'onde et de déterminer les coefficients d'extinction correspondant.

Les coefficients d'extinction ont été calculés par régression suivant les moindres rectangles (logiciel Systat 5.1 pour Macintosh, 1990-1991, Systat Inc). Pour la résolution des systèmes d'équation, un logiciel de résolution numérique a été utilisé (TK Solver Plus 1.1, version 2627, Universal Technical Systems Inc). L'erreur standard, ou écart type d'une valeur moyenne (Scherrer, 1984), est de nature statistique et caractérise la précision avec laquelle une valeur moyenne est déterminée. L'erreur absolue, de nature analytique, est la différence entre une valeur mesurée et la valeur théorique. Elle permet de caractériser la justesse d'un dosage. L'erreur relative est le quotient entre l'erreur absolue et la valeur théorique. Les répétitions incluent les phases de dilution et de dosage.

\section{RÉSULTATS ET DISCUSSION}

\section{Étude de l'ion nitrate}

\section{Caractéristiques spectrales}

L'absorbance de l'ion nitrate dans l'ultra-violet a été mise en évidence par de nombreux auteurs. Deux pics d'absorption sont observés, l'un à $300 \mathrm{~nm}$, l'autre vers $200 \mathrm{~nm}$ (Bastian et al, 1958 ; Hoather et Rackham, 1959 ; Cawse, 1967 ; Mac Donald et Haddad, 1970). Cependant, aux concentrations utilisées en solution nutritive, de l'ordre de la millimole par litre, l'absorbance à $300 \mathrm{~nm}$ n'est pas assez élevée pour être mesurée (absorbance spécifique de l'ordre de $10^{-6}$ ). L'étude montre l'existence d'un pic, dont la position, $201 \mathrm{~nm}$ (fig 1), et l'intensité, sont indépendantes du $\mathrm{pH}$ dans la gamme 4,5 à 7 et de la force ionique, jusqu'à $2510^{-3} \mathrm{~mol}^{-L^{-1}}$.

\section{Domaine de linéarité}

L'absorbance du nitrate a été étudiée aux différentes longueurs d'onde pour une gamme de

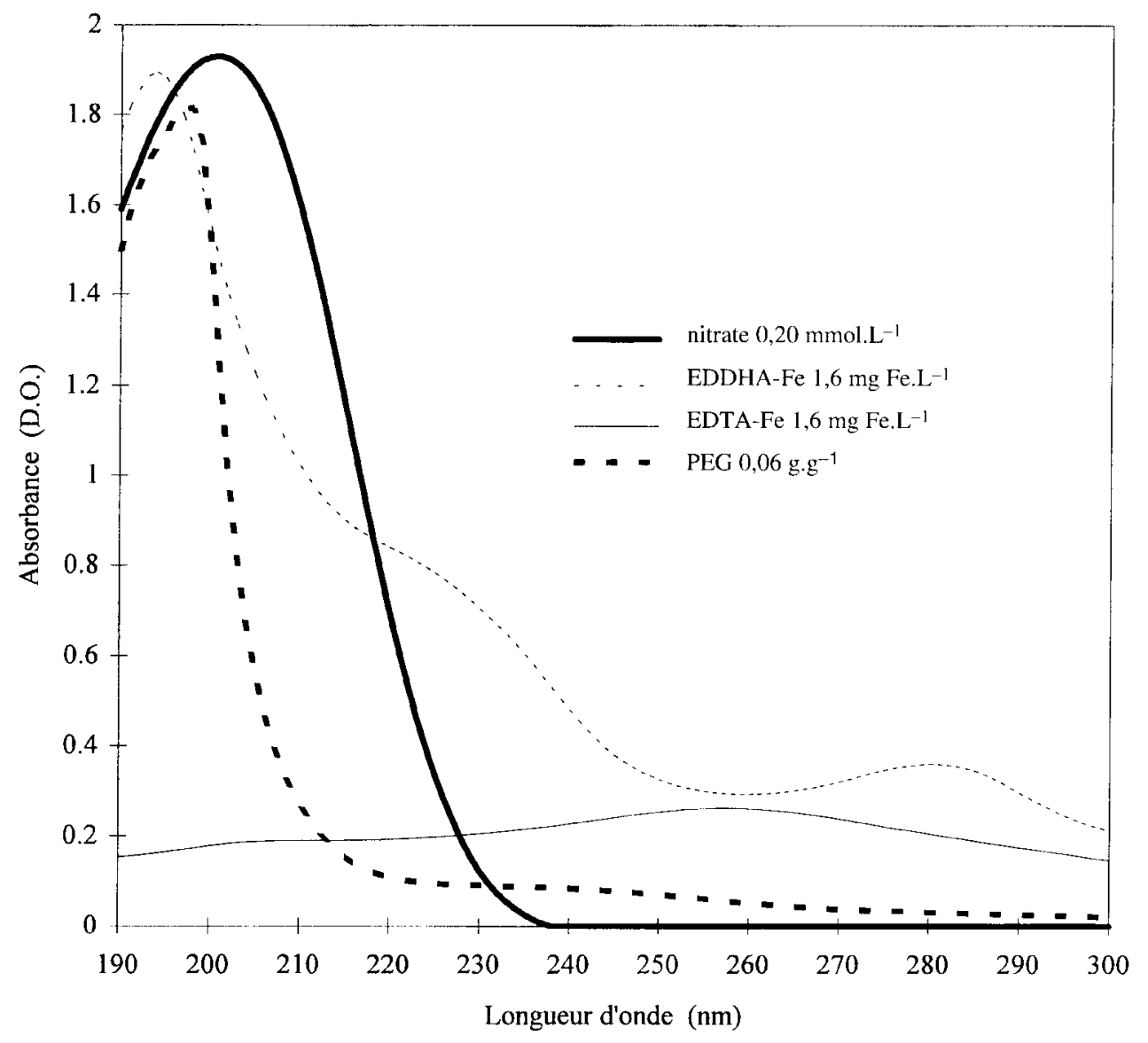

Fig 1. Spectre d'absorption de l'ion nitrate et des molécules interférentes aux concentrations indiquées. 
concentration s'étendant jusqu'à $0,510^{-3}$ mol.L-1. La loi de Beer-Lambert est suivie quelle que soit la longueur d'onde pour des valeurs inférieures à $0,210^{-3} \mathrm{~mol}^{-L^{-1}}$ (fig 2). Au-delà, l'étude des résidus de régression linéaire montre une nette courbure de la relation. Les coefficients d'extinction pour les différentes longueurs d'onde retenues sont présentés au tableau I.

\section{Étude de'T'EDDHA-Fe}

\section{Caractéristiques spectrales}

Le spectre de cette molécule, dans I'UV, présente deux pics d'absorption (vers $195 \mathrm{~nm}$ et $280 \mathrm{~nm}$ ) et un épaulement (fig 1), ainsi qu'un pic dans le visible vers $480 \mathrm{~nm}$, responsable de la
Fig 2. Relation entre absorbance et concentration en nitrate aux longueurs d'onde 201, 214 et $226 \mathrm{~nm}$ (quatre répétitions par concentration).

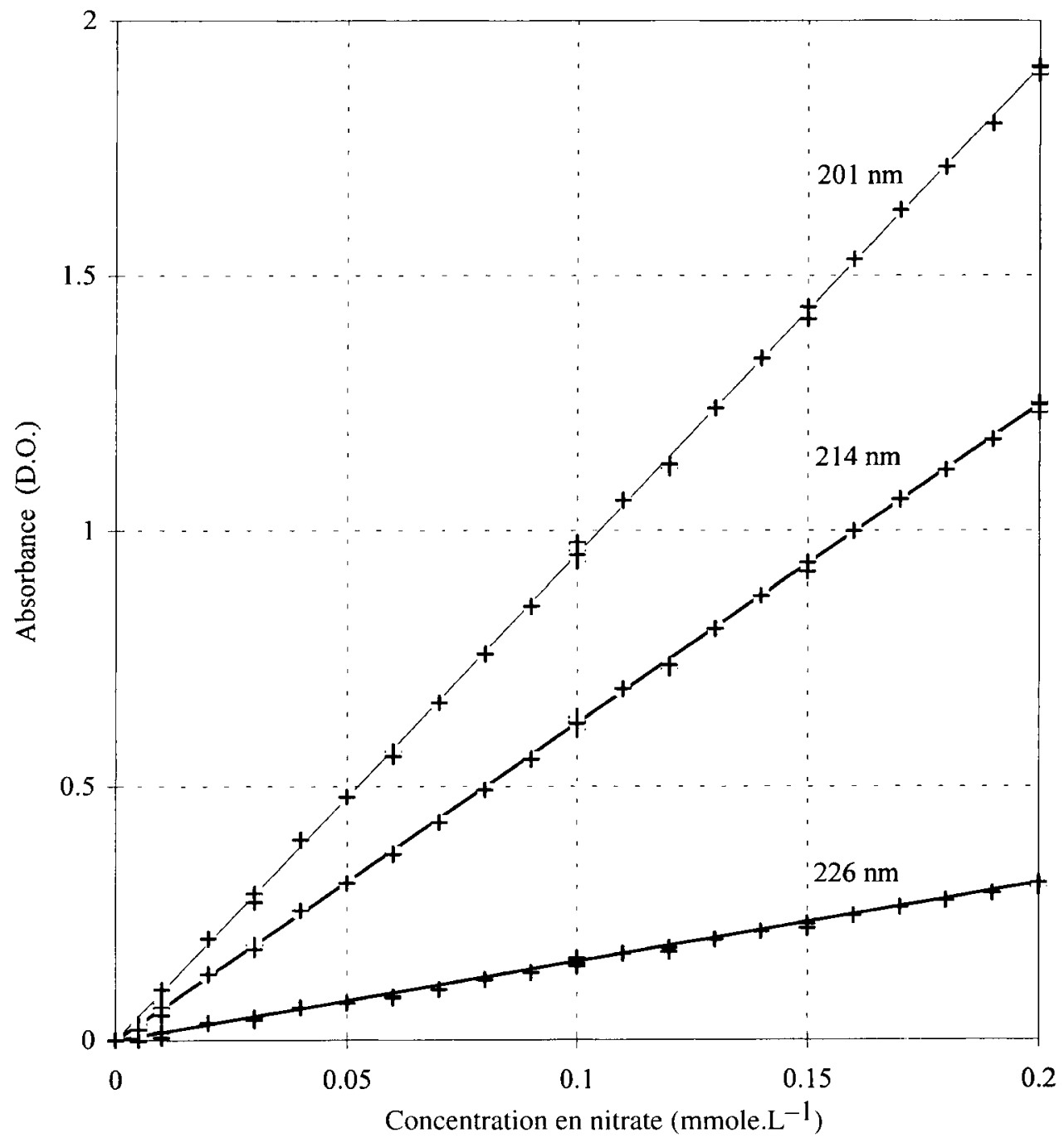

Tableau I. Coefficients d'extinction millimolaire $\varepsilon$ de l'ion nitrate et erreurs standard associées. Concentrations inférieures à $0,20 \mathrm{mmol} . \mathrm{L}^{-1}$, trajet optique de $1 \mathrm{~cm}$ et bande passante de $2 \mathrm{~nm}$.

Longueur d'onde $\lambda$

Coefficient d'extinction $\varepsilon$

$<0,001$

$<0,001$

1,7229

0,0005

Erreur type sur $\varepsilon$
$206 \mathrm{~nm}$

$201 \mathrm{~nm}$

6,6309

9,2513

9,6571

$\begin{array}{lll}- & 0,0005 & 0,0143\end{array}$

0,0293

0,0458 
couleur rouge de la solution. Le coefficient d'extinction de ce dernier pic, de l'ordre de 0,069 DO pour $1 \mathrm{mg} \mathrm{Fe} . \mathrm{L}^{-1}$, est cependant trop faible pour permettre une mesure précise de la concentration en séquestrène. L'étude de l'influence du milieu sur les caractéristiques spectrales du séquestrène montre que seule la position et le coefficient d'extinction du deuxième pic $(280 \mathrm{~nm})$ sont indépendants du $\mathrm{pH}$, alors que le coefficient d'extinction du premier pic (195 $\mathrm{nm}$ ) en est dépendant (fig 3). Toutefois, il existe un point isobestique (où l'absorbance n'est pas modifiée par le $\mathrm{pH}$ ) à $201 \mathrm{~nm}$, correspondant à une absorbance spécifique de 1,01 DO pour $1 \mathrm{mg} \mathrm{Fe} . \mathrm{L}^{-1}$. Le rapport de densité optique entre les pics à $195 \mathrm{~nm}$ et $280 \mathrm{~nm}$ varie fortement en fonction du $\mathrm{pH}$ (de 7,96 à pH 3,5 jusqu'à 5,56 à pH 7,0), alors qu'il est relativement stable entre $201 \mathrm{~nm}$ et $280 \mathrm{~nm}(4,485$ pour une gamme de $\mathrm{pH}$ allant de 4,6 à 7 , avec une erreur standard de 0,063). Ces deux dernières longueurs d'onde présentant les caractéristiques les moins variables, sont les plus favorables au dosage de I'EDDHA-Fe.

\section{Domaine de linéarité}

En se plaçant à $\mathrm{pH}$ 6, la linéarité de la réponse de l'absorbance à la concentration est vérifiée à toutes les longueurs d'onde $(195,201,206,254$, 280 et $482 \mathrm{~nm}$ ) jusqu'à une densité optique de 2,0 .

\section{Étude de I'EDTA-Fe-Na}

\section{Caractéristiques spectrales}

Le spectre de cette molécule, réalisé dans l'eau, montre l'existence de deux pics, tous deux dans I'UV (207 nm et $257 \mathrm{~nm}$ ). La couleur jaune clair de la solution correspond à la traînée dans le visible du pic à $257 \mathrm{~nm}$ (fig 1). Dans la gamme de $\mathrm{pH}$ étudiée, les caractéristiques spectrales (longueurs d'onde des maxima, hauteurs relatives et absolues des pics) sont constantes. L'absorbance de ces deux pics est de même

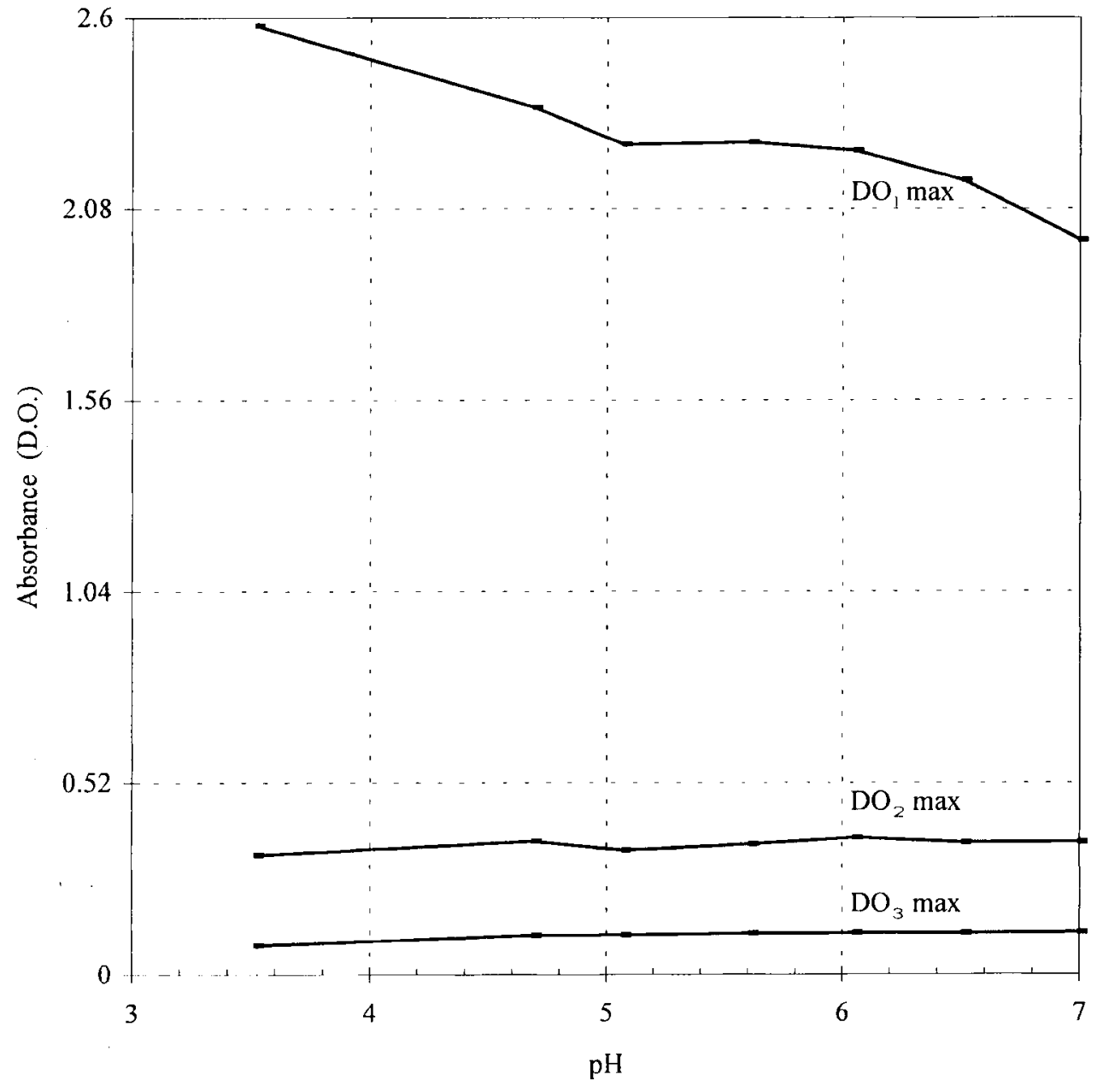

Fig 3. Effet du pH sur les trois maxima d'absorbance, indicés dans l'ordre croissant des longueurs d'onde, d'une solution d'EDDHA-Fe à une concentration équivalente à 1,6 mg Fe.L-1. 
ordre de grandeur, 0,116 à $207 \mathrm{~nm}$ et 0,148 à $257 \mathrm{~nm}$.

\section{Domaine de linéarité}

La relation absorbance-concentration a été étudiée dans une gamme allant jusqu'à $8 \mathrm{mg}$ Fe.L-1. La loi de Beer-Lambert est suivie à 201, 206 et $214 \mathrm{~nm}$ sur toute la gamme. En revanche à 257 et $280 \mathrm{~nm}$, l'étude des résidus montre une

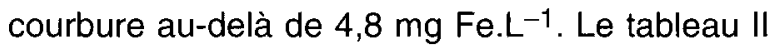
présente les coefficients d'extinction pour des concentrations en fer inférieures à cette dernière valeur.

\section{Étude d'autres formes d'EDTA}

Après absorption du fer par la plante, une inconnue existe sur le devenir du radical EDTA. Si cette molécule n'est pas absorbée, ce radical doit complexer d'autres cations, vraisemblablement du calcium pour l'essentiel. II importe donc également d'établir le spectre d'autres formes d'EDTA, pour évaluer le risque d'erreur analytique que pourrait faire courir leur accumulation. Pour des concentrations en molarité identiques, les spectres de trois formes d'EDTA ont été comparés (EDTA-Fe-Na, EDTA-Ca-Na ${ }_{2}$ et EDTA-Na 2 ). Les spectres sont profondément différents, et l'absorbance des autres formes d'EDTA est net- tement plus faible, les erreurs potentielles sont donc négligeables (tableau III).

\section{Étude du PEG6000}

\section{Caractéristiques spectrales}

L'étude du spectre du PEG6000 sur toute la gamme des longueurs d'onde montre un pic très net dans le lointain UV (fig 1). La longueur d'onde du maximum est située vers $198 \mathrm{~nm}$ et ce pic important chute brutalement jusqu'à $215 \mathrm{~nm}$, puis diminue très progressivement. Le $\mathrm{pH}$ n'a pas d'influence sur les caractéristiques spectrales du PEG6000.

\section{Domaine de linéarité}

La relation liant absorbance et concentration en PEG n'est pas linéaire, même pour de faibles concentrations (fig 4). Les régressions linéaires réalisées sur des gammes de concentrations restreintes montrent toujours une structuration nette dans la distribution des résidus. Cette non-linéarité constatée quelle que soit la longueur d'onde, et donc la gamme de densité optique, indique que ce ne sont pas les caractéristiques optiques du spectromètre qui sont en cause. Des tentatives de régressions non linéaires (ajustement de Le Bot, 1991, polynomiales, logarithmiques...

Tableau II. Coefficients d'extinction $\varepsilon$ de l'EDTA-Fe et erreurs standard associées. Concentrations inférieures à $4,8 \mathrm{mg}$ Fe $\mathrm{L}^{-1}$, trajet optique de $1 \mathrm{~cm}$ et bande passante de $2 \mathrm{~nm}$.

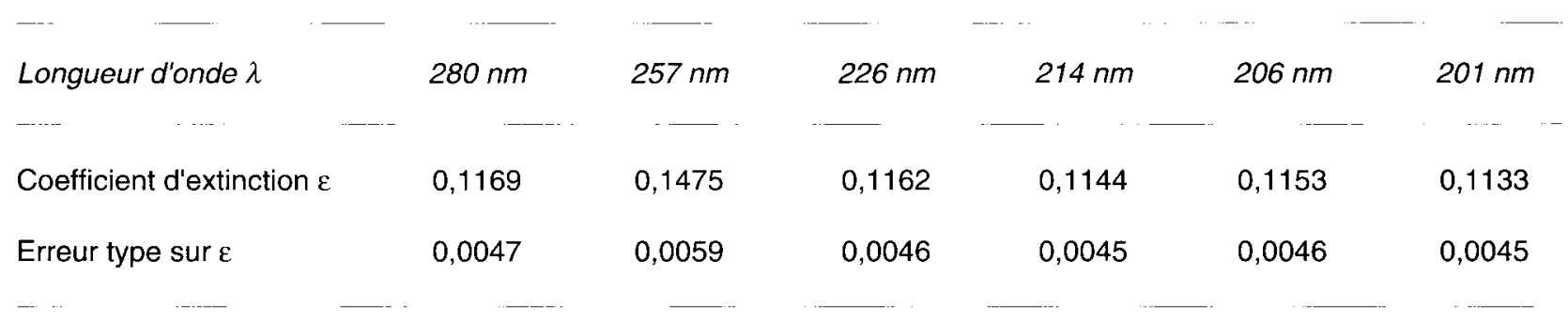

Tableau III. Équivalence en $\mu \mathrm{mol} . \mathrm{L}^{-1}$ d'ion nitrate, de divers chélates à une même concentration, correspondant pour I'EDTA-Fe et l'EDDHA-Fe à une valeur de $2,79 \mathrm{mg} \mathrm{Fe} \mathrm{L}^{-1}$.

$280 \mathrm{~nm} \quad 257 \mathrm{~nm}$

$226 \mathrm{~nm} \quad 214 \mathrm{~nm} \quad 206 \mathrm{~nm} \quad 201 \mathrm{~nm}$

EDTA-Fe en $\mu \mathrm{mol} . \mathrm{L}^{-1}$ équivalent $\mathrm{NO}_{3}^{-}$ EDTA-Ca en $\mu \mathrm{mol} . \mathrm{L}^{-1}$ équivalent $\mathrm{NO}_{3}^{-}$ EDTA-Na en $\mu$ mol. $L^{-1}$ équivalent $\mathrm{NO}_{3}^{-}$ Séquestrène en $\mu \mathrm{mol} . \mathrm{L}^{-1}$ éq $\mathrm{NO}_{3}^{-}$

$\begin{array}{llcccc}- & - & 211 & 50 & 35 & 32 \\ - & - & 0 & 2 & 7 & 11 \\ - & - & 0 & 0,4 & 2 & 3 \\ - & - & 1 & 1 & 260 & 325\end{array}$




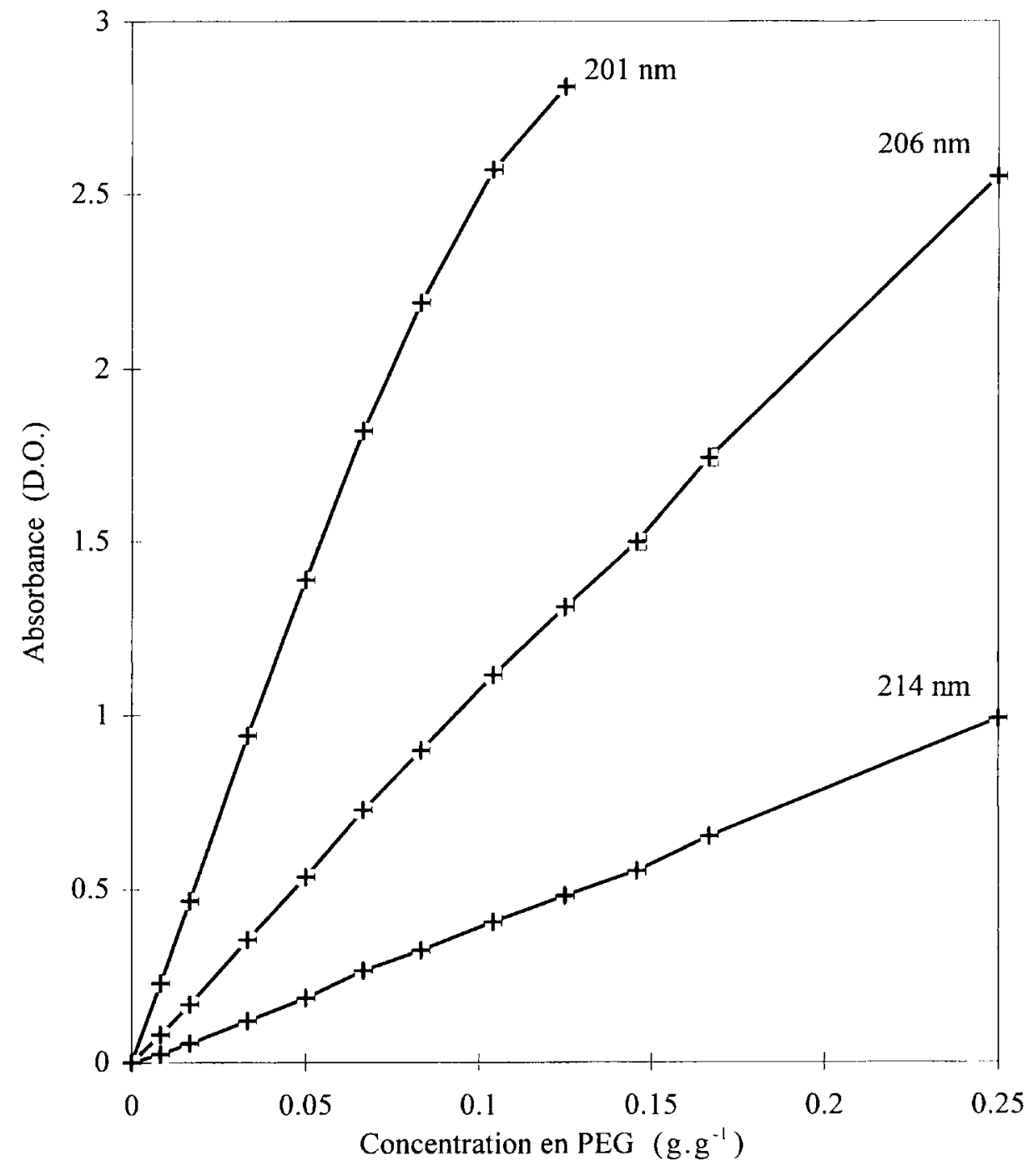

Fig 4. Relation entre absorbance et concentration en PEG aux Iongueurs d'onde 201, 206 et $214 \mathrm{~nm}$ (trois à quatre répétitions par concentration). n'ont pas permis d'aboutir à des ajustements satisfaisants présentant des résidus faibles et distribués de façon homogène. Le tableau IV présente les densités optiques moyennes, mesurées pour différentes concentrations en PEG. Entre ces points de gamme, les intrapolations linéaires ont été réalisées. Ces intrapolations donnent des ajustements plus satisfaisants que I'utilisation d'une régression non linéaire.

\section{Choix du chélate de fer}

Les deux chélates étudiés présentent une absorbance dans la bande d'absorption du nitrate, pouvant potentiellement fausser le dosage de ce dernier. L'utilisation de l'un ou de l'autre étant indifférente sur le plan de la nutrition minérale, il est possible de choisir le chélate le moins gênant pour un dosage précis de l'ion nitrate. Nous les comparons selon trois critères.

\section{Niveau d'interférence pour le dosage de l'ion nitrate}

Pour une même concentration finale en fer, l'absorbance de l'EDTA à $201 \mathrm{~nm}$ (correspondant au pic de l'ion nitrate) est dix fois plus faible que celle de l'EDDHA. Le tableau III montre qu'à une concentration de 2,8 $\mathrm{mg} \mathrm{Fe}^{-1} \mathrm{~L}^{-1}$ les absorbances de I'EDTA-Fe et de I'EDDHA-Fe sont équivalentes à 32 et $325 \mu \mathrm{mol} . \mathrm{L}^{-1}$ de nitrate respectivement.

\section{Incertitude induite sur le dosage de l'ion nitrate}

L'EDDHA et I'EDTA présentent chacun un deuxième pic au-delà de $250 \mathrm{~nm}$, domaine où le nitrate est transparent à l'UV. La mesure de l'absorbance à ces longueurs d'onde $(\lambda 1)$ permet de mesurer directement la concentration du chélate puis de calculer son absorbance dans le domai- 
Tableau IV. Absorbance du PEG6000 à plusieurs longueurs d'onde, en fonction de sa concentration exprimée en $\mathrm{g} . \mathrm{g}^{-1}$. Chaque donnée est issue de quatre répétitions. Valeurs en unités de densité optique, trajet optique de $1 \mathrm{~cm}$ et bande passante de $2 \mathrm{~nm}$.

\section{[PEG]}

$g \cdot g^{-1}$

0,008

0,017

0,033

0,050

0,067

0,083

0,104

0,125

0,146

0,167

0,250

\section{0,003 \\ 0,007}

0,014

0,024

0,057

0,048

0,060

0,074

0,077

0,115

0,143
0,004

0,010

0,022

0,037

0,070

0,071

0,089

0,108

0,119

0,157

0,220

Longueurs d'onde

$226 \mathrm{~nm} \quad 214 \mathrm{~nm}$

\section{0,010}

0,024

0,055

0,088

0,136

0,159

0,201

0,240

0,274

0,333

0,503

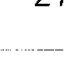

$\begin{array}{lll}0,024 & 0,079 & 0,228 \\ 0,054 & 0,168 & 0,465 \\ 0,120 & 0,354 & 0,941 \\ 0,186 & 0,534 & 1,388 \\ 0,265 & 0,727 & 1,819 \\ 0,323 & 0,898 & 2,189 \\ 0,405 & 1,115 & 2,569 \\ 0,481 & 1,310 & 2,811 \\ 0,551 & 1,498 & 2,959 \\ 0,651 & 1,741 & 3,224 \\ 0,990 & 2,551 & 3,427\end{array}$

ne de longueurs d'onde où le nitrate absorbe $\left(\lambda_{2}\right)$. La relation entre DO calculée $\left(\mathrm{DO}_{\lambda_{2}}\right)$ et $\mathrm{DO}$ mesurée $\left(\mathrm{DO}_{\lambda 1}\right)$ est de la forme :

$$
\mathrm{DO}_{\lambda 2}=\mathrm{DO}_{\lambda 1}{ }^{*} \varepsilon_{\lambda 2} / \varepsilon_{\lambda 1}
$$

II résulte que l'incertitude sur la contribution du chélate à l'absorbance dans le domaine de longueurs d'onde où le nitrate absorbé est proportionnelle à l'incertitude sur les mesures de densité optique multipliée par le rapport des coefficients d'extinction $\left(\varepsilon_{\lambda 2} / \varepsilon_{\lambda 1}\right)$. Dans le cas de I'EDDHA-Fe, ce rapport est de 4,3 entre 201 et $280 \mathrm{~nm}$, alors qu'il est de 0,77 pour l'EDTA-Fe entre 201 et $257 \mathrm{~nm}$.

\section{Sensibilité des caractéristiques spectrales aux conditions de milieu}

Contrairement à l'EDTA-Fe, les caractéristiques spectrales de l'EDDHA-Fe varient avec le $\mathrm{pH}$, ce qui pourrait contraindre à ajuster ce dernier à une valeur constante, ou à utiliser un tampon $\mathrm{pH}$. II reste cependant possible de se passer de cet artifice en mesurant les densités optiques à 201 et $280 \mathrm{~nm}$. Le rapport des coefficients d'extinction étant constant à ces longueurs d'onde quel que soit le $\mathrm{pH}$, la formule précédente montre que la contribution de l'EDDHA-Fe à l'absorbance à $201 \mathrm{~nm}$ peut être calculée. Toutefois, dans le cas de solutions nutritives contenant plus d'une molécule interférente pour le dosage de l'ion nitrate (chélate de fer et PEG par exemple), la nécessité d'utiliser au minimum trois longueurs d'onde n'autorise pas l'emploi d'EDDGA-Fe sans tamponner le $\mathrm{pH}$.
En conclusion, quel que soit le critère de comparaison, I'EDTA-Fe semble être le chélate le moins gênant pour le dosage de l'ion nitrate et, dans la suite de l'étude, nous l'avons donc retenu comme molécule complexante pour apporter le fer.

\section{Dosage de l'ion nitrate et de l'EDTA seuls}

Pour le dosage de l'ion nitrate en présence de molécules interférentes, il a été nécessaire de déterminer la combinaison de longueurs d'onde donnant la meilleure répétabilité pour la plus large gamme de concentration. La combinaison n'est pas forcément unique mais tient plutôt compte de la ou des molécules interférentes présentes en solution. Pour doser correctement le nitrate, il est nécessaire de doser également le plus justement possible les molécules interférentes présentes.

À chaque longueur d'onde, les erreurs standard sur la densité optique sont les mêmes pour le nitrate et pour l'EDTA-Fe. Ces erreurs, indépendantes du corps étudié, sont donc instrumentales (diluteur et spectromètre). De plus, les résidus sur la densité optique sont indépendants de la concentration (fig 5). Pour obtenir la meilleure précision relative, on diluera donc les échantillons pour obtenir une teneur en nitrate proche de $0,2010^{-3} \mathrm{~mol}^{-L^{-1}}$, concentration la plus élevée qui puisse être dosée sans s'écarter de la loi de Beer-Lambert.

Enfin, pour chaque ion, l'incertitude sur le dosage dépend aussi de la longueur d'onde choisie (tableau V). Sur toute la gamme des concentrations en EDTA ferrique, la meilleure justesse est obtenue à $280 \mathrm{~nm}$. 


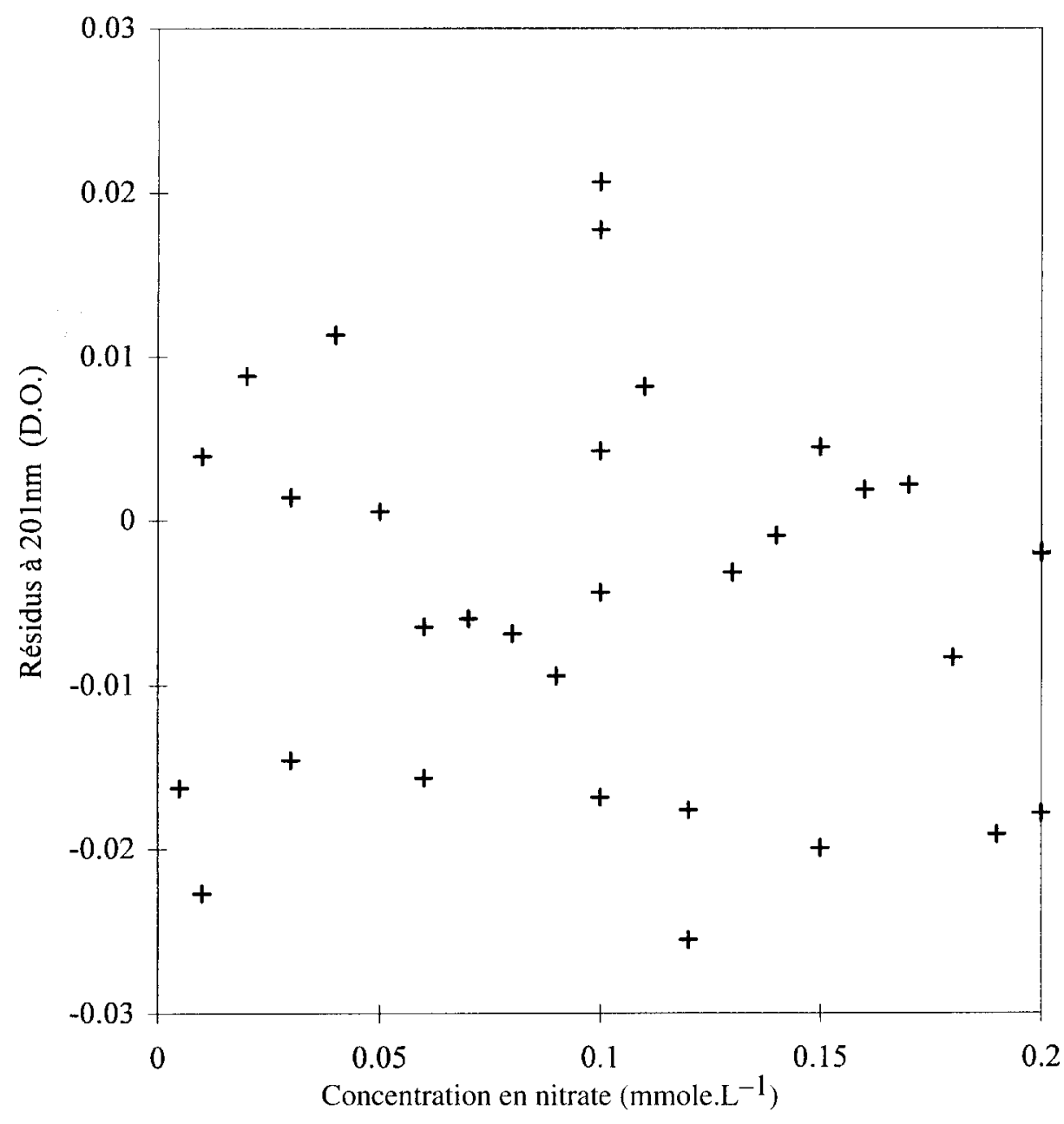

Fig 5. Valeurs des résidus de DO à $201 \mathrm{~nm}$ pour une gamme de concentration en nitrate.

\section{Dosage de l'ion nitrate en présence $d^{\prime} E D T A-F e$}

$$
\begin{aligned}
& \mathrm{DO}_{\lambda 1}^{\text {solution }}=\varepsilon_{\lambda 1}^{N O 3} \times\left[\mathrm{NO}_{3}\right]+\varepsilon_{\lambda 1}^{E D T A-\mathrm{Fe}} \times[\mathrm{Fe}] \\
& \mathrm{DO}_{\lambda 2}^{\text {solution }}=\varepsilon_{\lambda 2}^{\mathrm{NO} 3} \times\left[\mathrm{NO}_{3}\right]+\varepsilon_{\lambda 2}^{E D T A-\mathrm{Fe}} \times[\mathrm{Fe}]
\end{aligned}
$$

Avec une solution nutritive ne contenant comme molécule interférente que l'EDTA ferrique, le système se ramène à deux équations à deux inconnues, concentration en nitrate et en EDTA$\mathrm{Fe}$. Une lecture à deux longueurs d'onde suffit alors :

Nous avons fabriqué des solutions mélanges, avec des concentrations en nitrate allant de 0,10 à $510^{-3}$ mol. L- $\mathrm{L}^{-1}$ pour deux niveaux d'EDTA-Fe, 0,8 et $1,6 \mathrm{mg} \mathrm{Fe} . \mathrm{L}^{-1}$. Les mesures d'absorbance sur ces solutions montrent que les meilleures

Tableau V. Coefficients de variation sur les dosages de nitrate et d'EDTA-Fe en solutions pures, en fonction de leur

\begin{tabular}{|c|c|c|c|c|c|c|c|c|}
\hline \multirow{2}{*}{$\begin{array}{l}\text { Longueur } \\
\text { d'onde }\end{array}$} & \multicolumn{4}{|c|}{ Concentration en nitrate en $\mu \mathrm{mol} . \mathrm{L}^{-1}$} & \multicolumn{4}{|c|}{ Concentration en EDTA-Fe en mg Fe. $L^{-1}$} \\
\hline & 50 & 100 & 150 & 200 & 1,6 & 2,4 & 3,2 & 4,8 \\
\hline $201 \mathrm{~nm}$ & $1,7 \%$ & $0,4 \%$ & $0,5 \%$ & $0,1 \%$ & $6,9 \%$ & $3,3 \%$ & $2,5 \%$ & $1,0 \%$ \\
\hline $206 \mathrm{~nm}$ & $1,7 \%$ & $0,4 \%$ & $0,4 \%$ & $0,1 \%$ & $5,5 \%$ & $2,5 \%$ & $1,9 \%$ & $0,7 \%$ \\
\hline $214 \mathrm{~nm}$ & $2,2 \%$ & $0,4 \%$ & $0,3 \%$ & $0,02 \%$ & $2,3 \%$ & $0,8 \%$ & $0,7 \%$ & $0,3 \%$ \\
\hline $257 \mathrm{~nm}$ & - & - & - & - & $1,3 \%$ & $0,8 \%$ & $0,4 \%$ & $0,3 \%$ \\
\hline $280 \mathrm{~nm}$ & - & - & - & - & $0,1 \%$ & $0,4 \%$ & $0,01 \%$ & $0,5 \%$ \\
\hline
\end{tabular}
concentration et de la longueur d'onde (5 à 6 répétitions). 
combinaisons sont construites en choisissant une longueur d'onde dans chacun des deux binômes suivants, $201-206 \mathrm{~nm}$ et $257-280 \mathrm{~nm}$. Comme attendu, ces combinaisons font intervenir une longueur d'onde où l'absorption du nitrate est proche du maximum (201 ou $206 \mathrm{~nm}$ ) et une longueur d'onde où le nitrate est transparent (257 ou $280 \mathrm{~nm}$ ). La meilleure combinaison s'avère être 206-280 nm. Avec cette combinaison, l'erreur relative sur le dosage du nitrate est inférieure à $3 \%$. La répétabilité est également très bonne, le coefficient de variation étant largement inférieur à $1 \%$, et ceci pour l'ensemble de la gamme de concentration explorée, tant en nitrate qu'en EDTA-Fe. Les erreurs de justesse ne semblent pas dépendre de la concentration en EDTA ferrique, indiquant qu'il n'existe pas d'interaction entre le nitrate et l'EDTA. Pour doser le nitrate en présence d'EDTA ferrique en solution nutritive par spectroscopie UV, la seule contrainte est donc de diluer l'échantillon pour obtenir une concentration en nitrate comprise dans le domaine de linéarité de la loi de Beer-Lambert, et le plus proche possible de $0,2010^{-3} \mathrm{~mol}^{-\mathrm{L}^{-1}}$.

\section{Dosage de l'ion nitrate en présence d'EDTA-Fe et de PEG}

Théoriquement, dans les expérimentations, la concentration en PEG dans la solution est connue. Les expériences de physiologie végétale partent généralement de l'hypothèse que le PEG n'est pas absorbé par la plante. D'une part, ceci n'est peut être qu'une approximation, d'autre part, l'absorption d'eau par la plante induit une augmentation de la concentration en PEG qui peut se traduire par de très fortes variations d'absorbance pour les longueurs d'onde correspondant à la bande d'absorption du nitrate. De plus, la solution est rarement homogène pour le PEG, dont l'aptitude à mousser est une gêne pour pratiquer un brassage efficace au moment du prélèvement de l'échantillon. L'interférence correspond à l'absorbance calculée pour la concentration théorique en PEG, peut donc être très différente de l'interférence réelle et il importe donc de déterminer avec précision la contribution réelle du PEG à l'absorbance de la solution.

Pour une solution nutritive contenant du nitrate, du fer sous forme d'EDTA ferrique et du PEG, une lecture multi-longueurs d'onde de l'absorbance de la solution permet de calculer les concentrations des molécules interférentes, et par làmême d'en déduire la concentration du nitrate dans la solution :

$$
\begin{aligned}
\mathrm{DO}_{\lambda 1}^{\text {solution }}= & \varepsilon_{\lambda 1}^{N O_{3}} \times\left[N O_{3}\right]+\varepsilon_{\lambda 1}^{E D T A-F e} \times[F e] \\
& +f_{\lambda 1}^{P E G}([P E G]) \\
\mathrm{DO}_{\lambda 2}^{\text {solution }}= & \varepsilon_{\lambda 2}^{N O_{3}} \times\left[N O_{3}\right]+\varepsilon_{\lambda 2}^{E D T A-F e} \times[F e] \\
& +f_{\lambda 2}^{P E G}([P E G]) \\
\mathrm{DO}_{\lambda 3}^{\text {solution }}= & \varepsilon_{\lambda 3}^{N O_{3}} \times\left[N O_{3}\right]+\varepsilon_{\lambda 3}^{E D T A-F e} \times[F e] \\
& +f_{\lambda 3}^{P E G}([P E G])
\end{aligned}
$$

Pour le PEG6000, les valeurs de la fonction $f_{\lambda i}^{P E G}$ sont données par le tableau IV liant concentration et absorbance pour les différentes longueurs d'onde. Si nous avions trouvé une forme suffisamment approchée de cette fonction, il aurait été éventuellement possible de donner une solution algébrique à ce système d'équation, comme il a été fait pour des solutions contenant du nitrate et de I'EDTA-Fe. II a été jugé préférable de se référer au tableau IV et d'opérer des intrapolations linéaires entre les concentrations indiquées. C'est pourquoi nous avons développé une procédure de résolution numérique de ce système d'équation.

Nous avons fabriqué des solutions mélanges, avec des concentrations en nitrate allant de 0,08 à $1010^{-3} \mathrm{~mol}^{-L^{-1}}$ pour deux niveaux de $\mathrm{PEG}$ 0,12 et $0,17 \mathrm{~g} \cdot \mathrm{g}^{-1}$, correspondant respectivement à un potentiel hydrique de $-0,20$ et $-0,40 \mathrm{MPa}$. L'ensemble des combinaisons de longueurs d'onde a été exploré. Les combinaisons de longueurs d'onde comprenant 257 et $280 \mathrm{~nm}$ ont été écartées. En effet, le PEG absorbant très peu à ces longueurs d'onde, de faibles erreurs sur l'absorbance conduisent à de fortes erreurs sur la concentration calculée en PEG. Par suite, la contribution déduite du PEG à l'absorbance aux courtes longueurs d'onde peut donc potentiellement être très différente de la contribution réelle du PEG. La précision sur le dosage du nitrate en est d'autant moins bonne. Du fait de la très forte absorbance du PEG aux courtes longueurs d'onde (inférieures à $206 \mathrm{~nm}$ ), la somme des absorbances pour les différentes molécules en solution dépasse très rapidement les capacités optiques du spectromètre. Les combinaisons incluant $201 \mathrm{~nm}$ ont donc également été écartées. La combinaison 206-214-226 nm permet d'obtenir une très bonne précision sur la plus large gamme des concentrations explorées, tant en nitrate qu'en PEG. Lorsque les concentrations en nitrate 
sont élevées, la teneur en PEG dans la solution diluée est faible. Le dosage sur ces solutions donne alors d'excellents résultats. La précision des mesures est plus faible lorsque le rapport [PEG] $/\left[\mathrm{NO}_{3}\right]$ augmente, c'est-à-dire lorsque, pour une concentration faible en nitrate, la teneur en $P E G$ est élevée (fig 6). Pour des rapports inférieurs à 2 , la précision est meilleure que $3 \%$ et le coefficient de variation inférieur à $1 \%$. Pour un rapport égal à 1,4 , correspondant par exemple à une concentration en nitrate de $0,0810^{-3} \mathrm{~mol}^{-\mathrm{L}^{-1}}$ et à une teneur en PEG de $0,12 \mathrm{~g} \cdot \mathrm{g}^{-1}$, l'erreur relative moyenne est de $2,8 \%$. Ce rapport de composition semble pourtant, a priori, très défavorable à un dosage correct du nitrate. La contribution du nitrate à l'absorbance y est faible, sauf pour quelques longueurs d'onde (fig 7). Considérant que l'absorbance de l'EDTA est très faible, nous avons également étudié les résultats des dosages en éliminant la prise en compte de cette faible interférence. La résolution numérique du système aboutit souvent à une divergence, et lorsque les solutions existent, elles sont significativement moins précises. La combinaison rete- nue permet donc de doser correctement les trois molécules absorbantes de la solution. Au-delà de ce rapport de concentration, l'erreur relative augmente, ainsi que le coefficient de variation. La réponse du spectromètre n'est plus parfaitement linéaire pour des absorbances supérieures à 2 . Du fait de la forte absorbance du PEG pour les faibles longueurs d'onde, les densités optiques mesurées dépassent rapidement les capacités du spectromètre, même pour la combinaison de longueur d'onde retenue. Pour des niveaux d'absorbance limite, des abaques de concentrations en $P E G$ et en nitrate dans la solution ont été construits (fig 8). En se situant par exemple, au delà de la courbe absorbance $=2$, c'est-à-dire hors de la zone finement quadrillée, l'absorbance de la solution est supérieure au seuil choisi de 2 . II est donc nécessaire de diluer la solution pour se ramener dans cette zone, et l'on obtient alors des densités optiques admissibles au niveau d'absorbance choisi.

Pour le domaine de validité retenu, la seule contrainte inhérente à cette méthode est donc la dilution de la solution nutritive pour obtenir, d'une

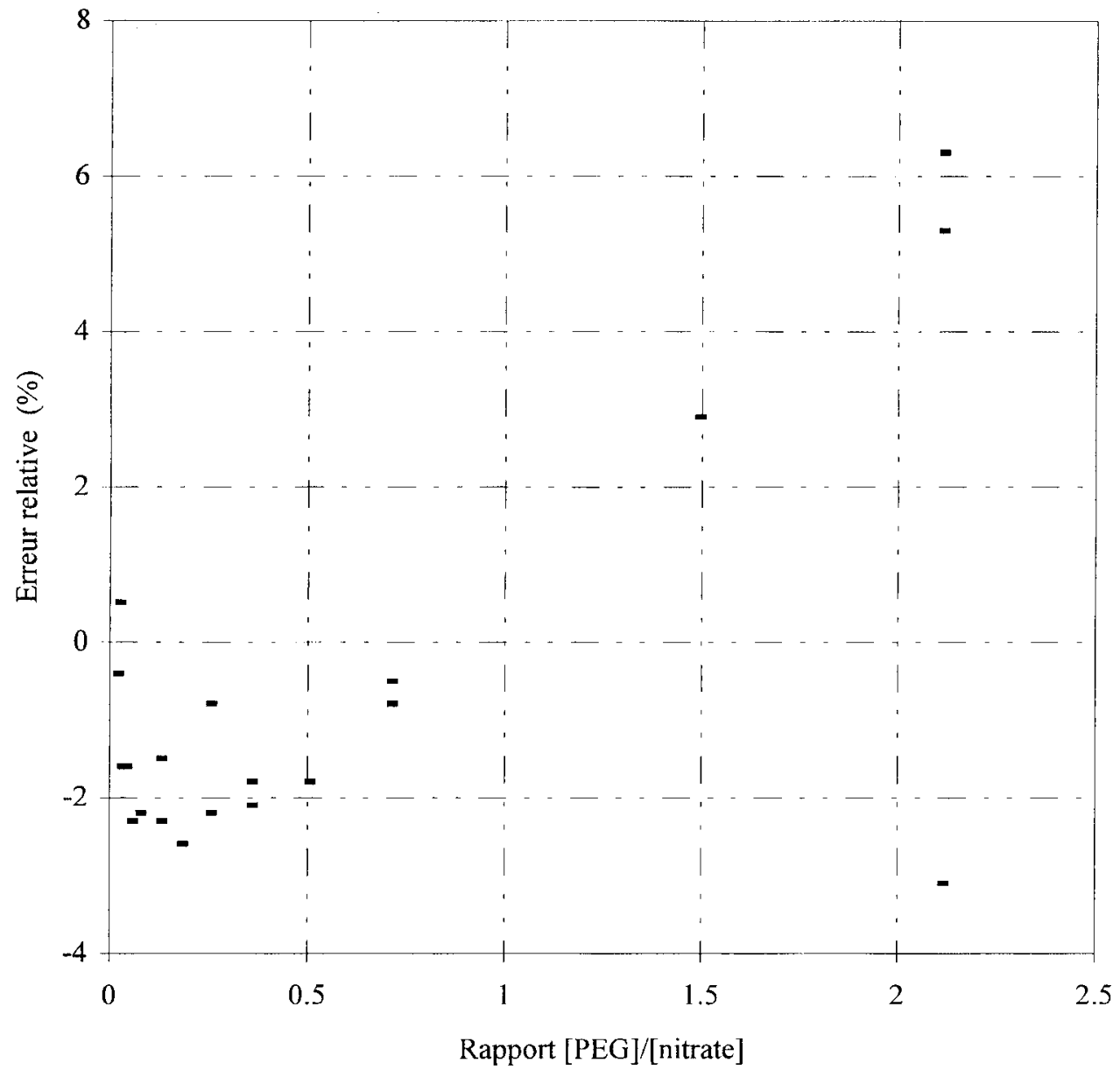

Fig 6. Valeurs des erreurs relatives moyennes (quatre répétitions) en fonction des rapports de concentration [PEG]/[nitrate]. Le PEG est exprimé en g.g-1 et le nitrate en mmol.L-1. 
Fig 7. Contribution des différentes molécules (nitrate, EDTA-Fe et PEG) en pourcentage de l'absorbance totale d'une solution, dans le cas d'un fort rapport $[\mathrm{PEG}] /[$ nitrate]

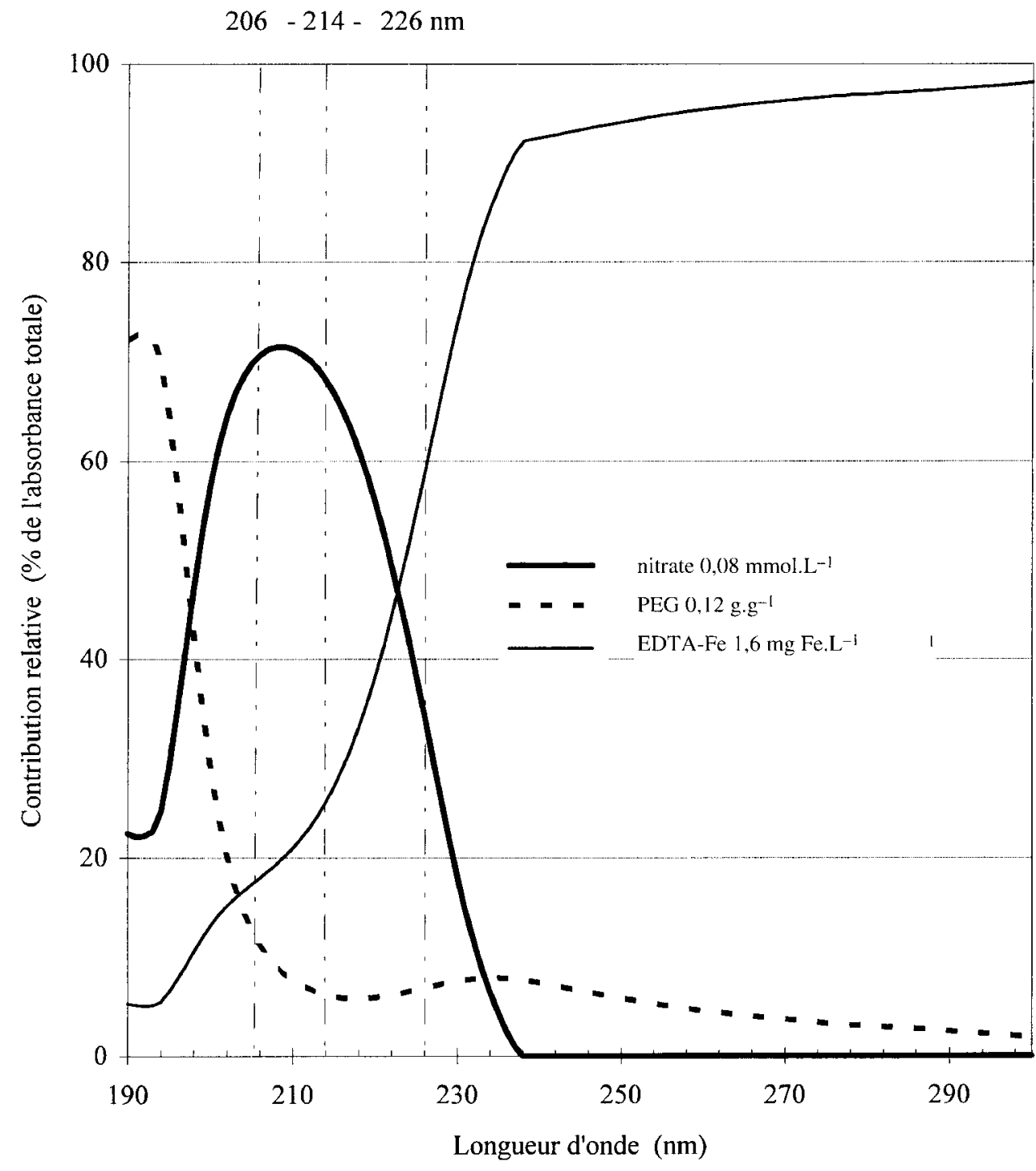

part, des concentrations en nitrate inférieures à $0,210^{-3} \mathrm{~mol}^{-\mathrm{L}^{-1}}$ (domaine de linéarité de la loi de Beer-Lambert pour le nitrate) et, d'autre part, des mesures d'absorbances inférieures à des valeurs critiques (domaine de linéarité du spectromètre).

\section{CONCLUSION}

La méthode exposée permet, en solution nutritive, le dosage du nitrate en le corrigeant des interférences. Très simple, elle ne nécessite pas d'ajout de réactif mais simplement une dilution de la solution pour obtenir une concentration nitrique inférieure à $0,2010^{-3} \mathrm{~mol}^{-L^{-1}}$. Elle est également très rapide (environ 1 minute par échantillon), et se prête à une automatisation simple, ce qui permet d'envisager aussi bien des dosages en continu que des dosages ponctuels.
Pour corriger le dosage du nitrate des interférences, les propriétés optiques des différentes molécules en solution ont été étudiées. Comme chélate de fer, l'EDTA-Fe a été retenu, son interférence étant faible (tableau III) et facilement prise en compte. Le comportement optique du PEG n'a pas permis d'obtenir des ajustements algébriques corrects. Des tables ont donc été construites pour les différentes longueurs d'onde retenues, sur lesquelles on peut opérer des ajustements linéaires par parties. Des mesures d'absorbance à plusieurs longueurs d'onde permettent de corriger le dosage du nitrate des interférences présentes. Pour une solution nutritive ne contenant que I'EDTA-Fe comme molécule interférante, la concentration en nitrate est obtenue en réalisant deux mesures d'absorbances :

$\left[\mathrm{NO}_{3}\right]=\frac{0,1169 \times \mathrm{DO}_{206 \mathrm{~nm}}^{\text {solution }}-0,1153 \times \mathrm{DO}_{280 \mathrm{~nm}}^{\text {solution }}}{1,0815}$ 


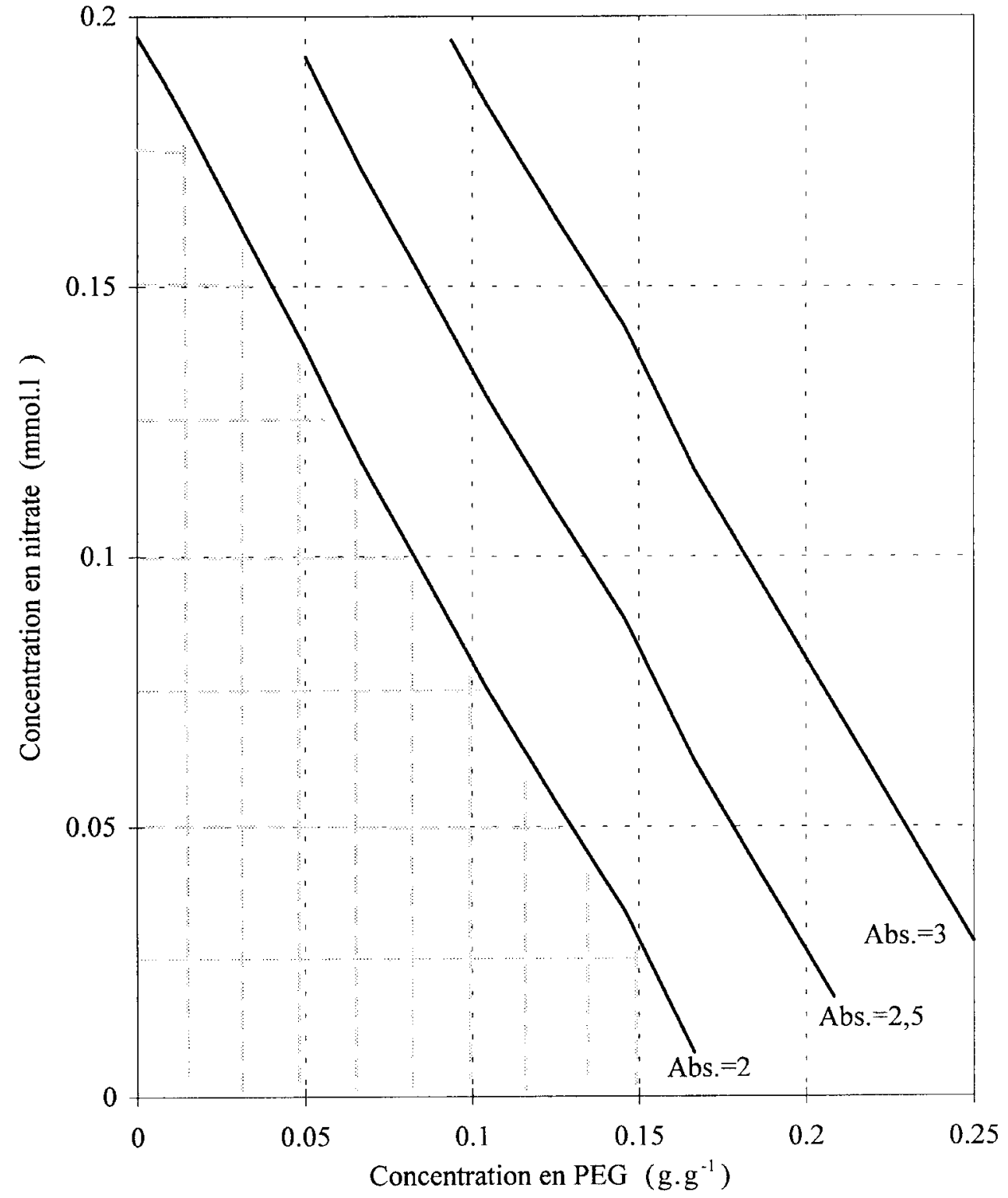

Fig 8. Courbes des niveaux d'absorbance totale (Abs) en fonction des teneurs en nitrate et en PEG, en présence d'EDTA-Fe (1,6 mg Fe.L $\left.\mathrm{L}^{-1}\right)$.
En présence de $P E G$, des mesures à trois longueurs d'onde sont nécessaires. La justesse du dosage est supérieure à $3 \%$ pour une très large gamme de concentration, ouvrant la possibilité de l'étude fine des problèmes de nutrition nitrique en condition de disponibilité hydrique réduite. Une comparaison des différentes méthodes de dosage du nitrate est actuellement également menée pour la validation, elle fera l'objet d'un prochain article. La méthode présentée a été utilisée au cours d'expériences agronomiques. Elle a permis de suivre une culture de tomates horssol, en solution recyclée sous serre, durant quatre mois, et a également permis d'étudier les liens existant entre l'absorption d'eau et l'absorption du nitrate chez le pêcher, dans des conditions de disponibilité hydrique limitante imposée par l'emploi de PEG.

\section{RÉFÉRENCES}

Adamowicz S, Otto C, Mars S, Ballino N (1982) Comparaison de différentes méthodes de dosage des nitrates dans les extraits de sols. Influence du milieu d'extraction. agronomie 2, 359-364

American Public Health Association (1965) Standard methods for the examination of water and waste water. APHA, New York, 195-204

Altshuller AP, Wartburg AF (1960) Ultraviolet determination of nitrogen dioxide as nitrite ion. Anal Chem 32, 174-177

Armstrong FAJ (1963) Determination of nitrate in water by ultraviolet spectrophotometry. Anal Chem 35 , 1292-1294

Baker AS, Smith R (1969) Extracting solution for potentiometric determination of nitrate in plant tissue. J Agric Food Chem 17,1284-1287 
Bastian R, Weberling R, Palilla F (1958) Ultraviolet spectrophotometric determination of nitrate. Application to analysis of alkaline earth carbonates. Anal Chem 29, 1795-1797

Breteler $H$, Hänisch Ten Cate $\mathrm{CH}$, Nissen P (1979) Time-course of nitrate uptake and nitrate reductase activity in nitrogen-depleted Dwarf Bean. Physiol Plant 47, 49-55

Breteler H, Nissen P (1982) Effect of exogenous and endogeneous nitrate concentration on nitrate utilisation by Dwarf Bean. Plant Physiol 70, 754-759

Buck RP, Samang Singhadeja, Rogers LB (1954) Ultraviolet absorption spectra of some inorganic ions in aqueous solutions. Anal Chem 26, 12401242

Cawse PA (1967) The determination of nitrate in soil solutions by ultraviolet spectrophotometry. Analyst $92,311-315$

Charlot G (1961) Dosages colorimétriques des éléments minéraux, principes et méthodes, $2^{\mathrm{e}}$ édition. Masson, Paris, $379 p$

Ciesielski $H$, Soignet $G$, Catoire M, Vancayzeele P (1978) Dosage colorimétrique des nitrates. Analusis $6,38-44$

Csiky I, Marko-Varga G, Jönsson JA (1985) Use of disposable clean-up columns for selective removal of humic substances prior to measurements with a nitrate ion-selective electrode. Anal Chim Acta 178, 307-312

Gholse SB (1984) Separation and microdetermination of nitrate with $N$ - $m$-tolyl- $p$-methoxy benzohydroxamic acid (TMBHA). J Indian Chem Soc 61, 467-468

Glover DJ, Hoffsommer JC (1974) Gas chromatography analysis of nitrate and nitrite ions in microgram quantities by conversion to nitrobenzene. $J$ Chromatography 94, 334-337

Heanes DL (1982) Determination of nitrate-N in plants by improved extractions procedure adapted for ultraviolet spectrophotometry. Commun Soil Sci Plant Anal 13, 803-818

Hoather RC, Rackham RF (1959) Oxidised nitrogen in waters and sewage effluents observed by ultraviolet spectrophotometry. Analyst 84, 548-551

Kamphake LJ, Hannah SA, Cohen JM (1967) Automated analysis for nitrate by hydrazine reduction. In: Water Research, Pergamon Press, Great Britain, 205-216

Keeney DR, Byrnes BH, Genson JJ (1970) Determination of nitrate in waters with the nitrateselective ion electrode. Analyst 95, 383-386

Lainé P, Ourry A, Macduff J, Boucaud J, Salette J (1993) Kinetic parameters of nitrate uptake by different catch crop species: effect of low temperatures or previous nitrate starvation. Physiol Plant 88, 85-92

Lambert RS, Du Bois RJ (1971) Spectrophotometric determination of nitrate in the presence of chloride. Anal Chem 43, 955-957

Le Bot J (1991) Diurnal uptake of nitrate and potassium by tomato (Lycopersicon esculatum Mill) plants. Thèse université Leeds, UK, $190 \mathrm{p}$
MacDonald JC, Haddad L (1970) Ultraviolet spectrophotometric determination of nitrite-nitrate in $\mathrm{KOH}$. Environ Sci Technol 4, 676-678

Michel BE (1983) Evaluation of the water potentials of solutions of polyethylene glycol 8000 built in the absence and presence of other solutes. Plant Physiol 72, 66-70

Ni M, Beevers $L$ (1990) Essential arginine residues in the nitrate uptake system from corn seedling roots. Plant Physiol 94, 745-751

Norwitz G, Keliher PN (1978) Inorganic interferences in the 2,4-xylenol spectrophotometric method for nitrate and their elimination. Anal Chim Acta 98, 323-333

Norwitz G, Farino J, Keliher PN (1979) Organic interferences and their elimination in the 2,4-xylenol spectrophotometric method for nitrate. Anal Chim Acta 105, 335-351

Novozamsky I, Houba VJG, van der Eijk D, van Eck R (1983) Notes on determinations of nitrate in plant material. Neth J Agric Sci 31, 239-248

Paul JL, Carlson RM (1968) Nitrate determination in plant extracts by the nitrate electrode. J Agric Food Chem 16, 766-768

Perrone A, Gant JR (1984) Ion chromatography using spectrophotometric detection. Applications study, Pittsburgh conference, paper 787, The Perkin Elmer Corporation, $23 p$

Pokorny G, Likussar W (1968) Use of oxazines in analysis. Determination of nitrate with Nil Blue A. Ann Chim Acta 42, 253-257

Quinche JP, Dvorak V (1980) Le dosage des nitrates dans les légumes, les plantes condimentaires et les terres par ionométrie et par chromatographie gazliquide. Revue Suisse Vitic Arboric Hortic 12, 7-20

Scherrer B (1984) Biostatistique. Morin, Chicoutimi, Québec, Canada, $850 p$

Smith KA, Scott A (1983) Continuous-flow and discrete analysis. In: Soil analysis. Instrumental Techniques and Related Procedures (KA Smith, ed), Books on Soil and the Environment, Marcel Dekker Inc, New York, 154-156

Snell FD, Snell CT (1949) Colorimetric methods of analysis, $3^{e}$ édition. D Van Nostrand Company, Inc, Toronto, $950 \mathrm{p}$

Talibudeen O, Page MB (1983) Ion selective electrodes. In: Soil analysis. Instrumental Techniques and Related Procedures (KA Smith, ed), Books on Soil and the Environment, Marcel Dekker Inc, New York, 55-113

West PW, Ramachandran TP (1966) Spectrophotometric determination of nitrate using chromotropic acid. Anal Chim Acta 35, 317-324

Williams RJ (1983) Determination of inorganic anions by ion chromatography with ultraviolet absorbance detection. Anal Chem 55, 851-854

Wu WS, Saschenbrecker PW (1977) Nitration of benzene as method for determining nitrites and nitrates in meat and meat products. J AOAC 60, 1137-1141 